\title{
VIBRO-ACOUSTIC ANALYSIS OF LAMINATED DOUBLE GLAZING USING THE FORCE IDENTIFICATION METHOD
}

\author{
Mounir Ben Jdidia, Ali Akrout, Dhouha Tounsi, Tahar Fakhfakh, \\ MOHAMED HADDAR \\ Mechanics, Modelling and Production Research Laboratory, Mechanical Engineering Department, National School \\ of Engineers of Sfax, Sfax, Tunisia \\ e-mail: mounir.benjdidia@isetsf.rnu.tn; ali.akrout@enit.rnu.tnandali_akrout2005@yahoo.fr; dhouha.ing@gmail.com; \\ tahar.fakhfakh@enis.rnu.tn; mohamed.haddar@enis.rnu.tn
}

\begin{abstract}
This paper presents a procedure for identifying wave forms and excitation frequencies of some forces applied on a given complex fluid-structure coupled system by using only its vibro-acoustic response. The considered concept is called the Independent Component Analysis (ICA) which is based on the Blind Source Separation (BSS). In this work, the ICA method is exploited in order to determine the excitation force applied to a thin-film laminated double glazing system enclosing a thin fluid cavity and limited by an elastic joint. The dynamic response of the studied fluid-structure coupled system is determined by finite element discretization and minimization of the homogenized energy functional of the coupled problem. This response will serve as the input for the ICA algorithm in order to extract the applied excitation.
\end{abstract}

Keywords: fluid-structure interaction, thin-film laminated plate, ICA, excitation force, Kurtosis

\section{Introduction}

Laminated and sandwich plates present their advantage when they are used to reduce noise and vibration due to their high structural damping. In fact, the presence of viscoelastic core or ultra-thin film gives more rigidity to laminates with a much reduced vibration deflection.

That is why the resolution of direct fluid-structure coupled problems, such as the study of acoustic transparency of double panel systems, has been carried out in several research works such as those developed by Cheng et al. (2005), Abdennadher et al. (2005) and Akrout et al. (2010).

In this work, we deal with a system composed of a thin-film laminated double glazing system enclosing a thin fluid cavity and limited by an elastic joint, to which we use the ICA concept in order to extract the applied force.

In fact, the Blind Source Separation (BSS) is an important research area in signal processing and data analysis.

The first formulation of the problem was made in 1985 by researchers in neuroscience and signal processing to model biologically coding of motion. In fact, the source separation problem was developed by Hérault and Ans (1984) and Hérault et al. (1985). Then, Comon (1994) made the link between the Independent Component Analysis (ICA) and the Blind Source Separation (BSS).

The independent component analysis (ICA) is one of the major pathways of sources separation concept (Hyvärinen and Oja, 2000; Antoni, 2005; Zarzoso and Comon, 2008; Abbes et al., 2011; Akrout et al., 2012b). It extracts from the observed signal components as independent as possible. In the recent years, this method (ICA) was investigated for extracting signals such as 
excitation forces and internal defaults in mechanical systems (Akrout et al., 2012b; Taktak et al., 2012).

In this work, dynamical study is carried out in order to model the vibratory excitation of a vibro-acoustic problem defined by a thin fluid cavity coupled to the thin-film laminated glass plate structural model developed in our previous work (Akrout et al., 2012a). Then, the ICA concept is applied to the finite element signals defined by the displacement vector of the studied system in order to extract the wave form and the excitation frequencies of the external applied forces. So, the main original contribution of this work is based on the developed fluid-structure laminated double glazing model which can be exploited for identifying excitation sources by the inverse method (ICA).

\section{Description of the studied fluid-structure coupled system}

The studied system is composed of two ultra-thin film laminated glass plates coupled to a thin fluid cavity and related with an elastic joint, as presented in Fig. $1 . k_{0}, k_{1}$ and $k_{2}$ represent the laminate edge stiffness and $F_{0}$ is the harmonic uniform distributed force applied to thin-film laminated plate 1.

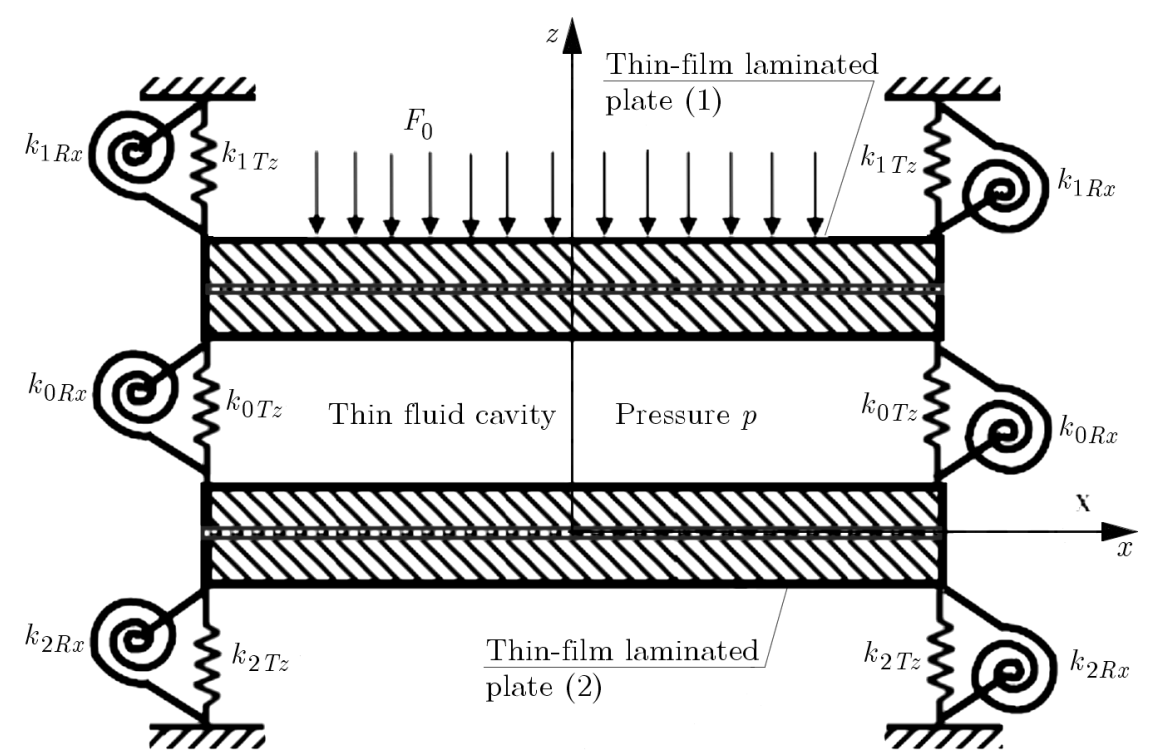

Fig. 1. Ultra-thin film laminated double glazing system (the $x z$-plane)

\section{Resolution of the direct vibro-acoustic problem}

\subsection{Dynamic equation of the coupled problem}

Discretization by the finite element method and minimization of the coupled system energy functional give the following coupled matrix system (Akrout et al., 2010, 2012a)

$$
\left[\begin{array}{ccc}
\mathbf{K}_{1}+\mathbf{J}_{1}+\mathbf{J}_{0}-\omega^{2} \mathbf{M}_{1} & -\mathbf{J}_{012} & -\mathbf{C}_{1} \\
-\mathbf{J}_{012}^{\mathrm{T}} & \mathbf{K}_{2}+\mathbf{J}_{2}+\mathbf{J}_{0}-\omega^{2} \mathbf{M}_{2} & \mathbf{C}_{2} \\
-\mathbf{C}_{1}^{\mathrm{T}} & \mathbf{C}_{2}^{\mathrm{T}} & \frac{h_{f}}{\rho_{f} \omega^{2}}\left(\mathbf{H}-k_{f}^{2} \mathbf{Q}\right)
\end{array}\right]\left\{\begin{array}{c}
\mathbf{U}_{1} \\
\mathbf{U}_{2} \\
\mathbf{P}
\end{array}\right\}=\left\{\begin{array}{c}
\mathbf{F}^{e x t} \\
\mathbf{0} \\
\mathbf{0}
\end{array}\right\}
$$

where $\mathbf{K}_{1}+\mathbf{J}_{1}+\mathbf{J}_{0}, \mathbf{K}_{2}+\mathbf{J}_{2}+\mathbf{J}_{0}$ and $\mathbf{J}_{012}$ are the stiffness matrices of the structural part of the coupled system. $\mathbf{M}_{1}$ and $\mathbf{M}_{2}$ are the mass matrices of the laminates. $\mathbf{C}_{1}$ and $\mathbf{C}_{2}$ are the fluid- 
-structure coupling matrices. $\mathbf{H}$ and $\mathbf{Q}$ represent the acoustic matrices. $h_{f}, \rho_{f}$ and $k_{f}=\omega / c_{f}$ represent the cavity thickness, the fluid density and the acoustic wave number, respectively. $c_{f}$ and $\omega$ are the speed of sound and the angular frequency, respectively. $\mathbf{U}_{1}, \mathbf{U}_{2}$ and $\mathbf{P}$ are the nodal response vectors. $\mathbf{F}^{e x t}=\mathbf{F}_{0} \exp (-\mathrm{i} \omega t)$ represent the nodal force vector to be reconstituted by the developed inverse method (ICA).

The film-laminate finite element presents seven degrees of freedom at each node $\left(u_{m}, v_{m}, \mathbf{u}_{\tau}, \mathbf{v}_{\tau}, w, \beta_{x}, \beta_{y}\right)$, whereas the fluid cavity finite element presents one degree of freedom at each node (pressure $p$ ).

\subsection{Resolution of the dynamic equation}

The resolution of the direct problem defined by equation (3.1) is based on a modal approach (Akrout et al., 2010, 2012a). Then, the following eigenvalue problems to be solved are considered

$$
\begin{array}{ll}
\left(\mathbf{K}_{1}+\mathbf{J}_{1}+\mathbf{J}_{0}-\omega^{2} \mathbf{M}_{1}\right) \mathbf{U}_{1}=\mathbf{0} & \left(\mathbf{K}_{2}+\mathbf{J}_{2}+\mathbf{J}_{0}-\omega^{2} \mathbf{M}_{2}\right) \mathbf{U}_{2}=\mathbf{0} \\
\frac{h_{f}}{\rho_{f} \omega^{2}}\left(\mathbf{H}-k_{f}^{2} \mathbf{Q}\right) \mathbf{P}=\mathbf{0} &
\end{array}
$$

By resolving these equations, three modal bases could be constructed: $\boldsymbol{\Phi}_{s 1}$ for the structure defined by (laminate $1+$ joint $1+$ joint 0$), \boldsymbol{\Phi}_{s 2}$ for the structure (laminate $2+$ joint $2+$ joint 0 ) and $\boldsymbol{\Phi}_{f}$ for the fluid cavity. The second step consists on reducing the size of system (3.1) by modal projection on these modal bases. So, matrix system (3.1) becomes (Akrout et al., 2010, 2012a)

$$
\left[\begin{array}{ccc}
\left(\overline{\mathbf{K}}_{1}+\overline{\mathbf{J}}_{1}+\overline{\mathbf{J}}_{0}\right)-\omega^{2} \overline{\mathbf{M}}_{1} & -\overline{\mathbf{J}}_{012} & -\overline{\mathbf{C}}_{1} \\
-\overline{\mathbf{J}}_{012}^{\mathrm{T}} & \left(\overline{\mathbf{K}}_{2}+\overline{\mathbf{J}}_{2}+\overline{\mathbf{J}}_{0}\right)-\omega^{2} \overline{\mathbf{M}}_{2} & \overline{\mathbf{C}}_{2} \\
-\overline{\mathbf{C}}_{1}^{\mathrm{T}} & \overline{\mathbf{C}}_{2}^{\mathrm{T}} & \frac{h_{f}}{\rho_{f} \omega^{2}}\left(\overline{\mathbf{H}}-k_{f}^{2} \overline{\mathbf{Q}}\right)
\end{array}\right]\left\{\begin{array}{c}
\overline{\mathbf{U}}_{1} \\
\overline{\mathbf{U}}_{2} \\
\overline{\mathbf{P}}
\end{array}\right\}=\left\{\begin{array}{c}
\overline{\mathbf{F}}^{e x t} \\
\mathbf{0} \\
\mathbf{0}
\end{array}\right\}
$$

where $\left(\overline{\mathbf{K}}_{i}+\overline{\mathbf{J}}_{i}+\overline{\mathbf{J}}_{0}\right)=\boldsymbol{\Phi}_{s i}^{\mathrm{T}}\left(\mathbf{K}_{i}+\mathbf{J}_{i}+\mathbf{J}_{0}\right) \boldsymbol{\Phi}_{s i}, \overline{\mathbf{M}}_{i}=\boldsymbol{\Phi}_{s i}^{\mathrm{T}} \mathbf{M}_{i} \boldsymbol{\Phi}_{s i}(i=1,2)$ are respectively the reduced stiffness and mass matrices of the coupled system. $\overline{\mathbf{J}}_{012}=\boldsymbol{\Phi}_{s 1}^{\mathrm{T}} \mathbf{J}_{012} \boldsymbol{\Phi}_{s 2}$ is the reduced stiffness matrix due to the structural coupling between laminate 1 and $2 . \overline{\mathbf{H}}=\boldsymbol{\Phi}_{f}^{\mathrm{T}} \mathbf{H} \boldsymbol{\Phi}_{f}$ and $\overline{\mathbf{Q}}=\boldsymbol{\Phi}_{f}^{\mathrm{T}} \mathbf{Q} \boldsymbol{\Phi}_{f}$ represent the reduced acoustic matrices. $\overline{\mathbf{C}}_{i}=\boldsymbol{\Phi}_{s i}^{\mathrm{T}} \mathbf{C}_{i} \boldsymbol{\Phi}_{f}$ is the reduced fluid-laminate $(i=1,2)$ coupling matrix. $\overline{\mathbf{U}}_{1}=\boldsymbol{\Phi}_{s 1}^{\mathrm{T}} \mathbf{U}_{1}, \overline{\mathbf{U}}_{2}=\boldsymbol{\Phi}_{s 2}^{\mathrm{T}} \mathbf{U}_{2}$ and $\overline{\mathbf{P}}=\boldsymbol{\Phi}_{f}^{\mathrm{T}} \mathbf{P}$ represent the modal response vectors and $\overline{\mathbf{F}}^{e x t}=\boldsymbol{\Phi}_{s 1}^{\mathrm{T}} \mathbf{F}^{e x t}$ is the modal force vector.

The coupled modal basis $\boldsymbol{\Phi}_{c}=\left[\phi_{1}, \ldots, \phi_{N}\right]$ ( $N$ is the number of retained eigenmodes) which contains the coupled eigenmodes, is obtained from the resolution of the eignemode reduced coupled problem as presented in our previous works (Akrout et al., 2010, 2012a).

The modal variables $\alpha_{r}(\omega)$ of the $r$-th eigenmode, which are obtained by projecting equation (3.5) on the coupled eigenmode basis $\boldsymbol{\Phi}_{c}$, could have the following expression (Akrout et al., 2010)

$$
\alpha_{r}(\omega)=\frac{f_{r}}{k_{r}}\left[1-\left(\frac{\omega}{\omega_{r}}\right)^{2}\right]^{-1} \quad r=1,2, \ldots, N
$$

where $\omega_{r}=\sqrt{k_{r} / m_{r}}$ is the $r$-th eigenfrequency of the coupled system. $k_{r}$ and $m_{r}$ are the $r$-th generalized mass and the $r$-th generalized stiffness of the coupled system, respectively. $f_{r}$ is the generalized force of the $r$-th eignmode.

So, the dynamic response is determined by modal recombination (Hammami et al., 2005; Akrout et al., 2010)

$$
\mathbf{U}(\omega)=\left[\phi_{1}, \ldots, \phi_{N}\right]\left\{\begin{array}{c}
\alpha_{1}(\omega) \\
\vdots \\
\alpha_{N}(\omega)
\end{array}\right\}
$$


Now, after determining the dynamic response which is defined by the displacement of the studied structure, we will study the inverse problem. In this case, the dynamic response will serve as the input for the ICA algorithm in order to determine the wave form of the applied force signal.

\section{The concept of the Independent Component Analysis: ICA}

\subsection{Definition and principle hypothesis}

ICA is a statistical technique that aims to break a random signal multivariate $X$ (measured signal) in a multivariable linear combination of independent signals (the source signals) to highlight the signals as independent as possible from the measured signals.

It was developed by Hérault et al. (1985) and it is defined by the following equation

$$
\mathbf{X}(t)=\mathbf{A} \mathbf{S}(t)
$$

where $\mathbf{X}(t)$ and $\mathbf{S}(t)$ are respectively the observed signals through sensors and the source signals, $\mathbf{A}$ is the mixture matrix.

The source separation principle consists of determining a matrix $\mathbf{B}$ in order to estimate $N$ source signals defined by the vector $\mathbf{Y}(t)=\left[Y_{1}(t), \ldots, Y_{N}(t)\right]^{\mathrm{T}}$ as follows (Antoni, 2005; Abbès et al., 2011; Akrout et al., 2012b)

$$
\mathbf{Y}(t)=\mathbf{B X}(t)
$$

In order to achieve this goal, general assumptions must be considered (Hyvärinen and Oja, 2000). The principal assumption is the statistical independency of the source signals. The second one imposes non-Gaussian distributions on the source signals (Comon, 1991; Moreau and Macchi, 1993). The last one is defined by the principle of uncorrelated sources. In fact two variables $Y_{1}$ and $Y_{2}$ are uncorrelated if their covariance is equal to zero. This can be expressed by the following relation

$$
E\left\{Y_{1} Y_{2}\right\}-E\left\{Y_{1}\right\} E\left\{Y_{2}\right\}=0
$$

\subsection{Separation concept}

The principle object of the presented method is to extract the source signals from a mixture of the observed signals. In order to achieve this goal, the observed signals of the system should be centred (substrate its mean vector), then whitened (which consist on eliminating the noise, so we obtain a new signal with an uncorrelated component and variance equal to unity). So, the estimated source is defined by Antoni (2005)

$$
\mathbf{Y}=\mathbf{W}^{\mathrm{H}} \mathbf{X}
$$

where $\mathbf{W}^{\mathrm{H}}$ is the separating matrix, $(\cdot)^{\mathrm{H}}$ denotes the conjugate-transpose operator.

In order to guarantee the non-gaussianity of the signals, the estimated sources must maximize the contrast function. The contrast function utilized in the ICA algorithm is the Kurtosis function defined by Zarzoso and Comon (2010). This forth order cumulant can be normalized as follows

$$
K(\omega)=\frac{E\left\{|y|^{4}\right\}-2 E^{2}\left\{|y|^{2}\right\}-\left|E\left\{y^{2}\right\}\right|^{2}}{E^{2}\left\{|y|^{2}\right\}}
$$

Finally, after determining the first column of the separating matrix, the deflation approach is applied in order to extract the corresponding source vector from the original mixture related to the determined column of the separating matrix.

So, each source will be chosen once with the multiplying factor (Hyvärinen and Oja, 2000). 


\section{Numerical results}

The geometrical and physical features of the ultra-thin film laminated glass panel are given as follows (Akrout et al., 2010):

- two identical skins (glass) for each laminate:

- Young's modulus $E_{1}^{p 1}=E_{2}^{p 1}=E_{1}^{p 2}=E_{2}^{p 2}=7.2 \cdot 10^{4} \mathrm{MPa}$

- density $\rho_{1}^{p 1}=\rho_{2}^{p 1}=\rho_{1}^{p 2}=\rho_{2}^{p 2}=2500 \mathrm{Kg} / \mathrm{m}^{3}$

- Poisson's ratios $\nu_{1}^{p 1}=\nu_{2}^{p 1}=\nu_{1}^{p 2}=\nu_{2}^{p 2}=0.22$

- skin thicknesses for the symmetrical system $h_{1}^{p 1}=h_{2}^{p 1}=h_{1}^{p 2}=h_{2}^{p 2}=3 \mathrm{~mm}$

- skin thicknesses for the asymmetrical system $h_{1}^{p 1}=h_{2}^{p 1}=3 \mathrm{~mm}, h_{1}^{p 2}=h_{2}^{p 2}=4 \mathrm{~mm}$

- film stiffness $k_{f i l m}=1.362 \cdot 10^{7} \mathrm{~N} / \mathrm{mm}^{3}$ (Araldite), $k_{f i l m}=1.1 \cdot 10^{4} \mathrm{~N} / \mathrm{mm}^{3}$ (Epoxy)

- in-plane $(x, y)$ laminate half dimensions $\ell_{x}=0.6 \mathrm{~m}, \ell_{y}=0.4 \mathrm{~m}$ '

The geometrical and physical parameters of the air cavity are given as follows (Akrout et al., 2010): $c_{f}=340 \mathrm{~m} / \mathrm{s}, h_{f}=1 \mathrm{~mm}, \rho_{f}=1.2 \mathrm{Kg} / \mathrm{m}^{3}$.

Only the translational joint is considered (Akrout et al., 2012a): $k_{\text {joint }}=0.264 \cdot 10^{10} \mathrm{~N} / \mathrm{mm}^{2}$.

\subsection{Parametric study}

Three configurations for the coupled studied system are considered:

- model 1 defined by two identical laminates $\left(h_{1}^{p 1}=h_{2}^{p 1}=h_{1}^{p 2}=h_{2}^{p 2}=3 \mathrm{~mm}\right)$ with an ultra-thin film of Araldite $\left(k_{\text {film }}=1.362 \cdot 10^{7} \mathrm{~N} / \mathrm{mm}^{3}\right)$

- model 2 characterized by the same laminates as model 1 , but another material is chosen for the adhesive film $\left(k_{\text {film }}=1.1 \cdot 10^{4} \mathrm{~N} / \mathrm{mm}^{3}\right.$, Epoxy $)$

- model 3 distinguished by an asymmetrical double glazing system (laminate $1: h_{1}^{p 1}=h_{2}^{p 1}=$ $3 \mathrm{~mm}$, laminate $\left.2: h_{1}^{p 2}=h_{2}^{p 2}=4 \mathrm{~mm}, k_{f i m}=1.362 \cdot 10^{7} \mathrm{~N} / \mathrm{mm}^{3}\right)$.

For these three different models, two types of loads are applied on laminate 1: the first one is defined by a uniformly distributed force and the second one is a punctual force.

\subsection{Observed signals}

For each model defined above, the FE coupled system vibratory responses (transversal displacement on the middle of the first and second laminate: $\left.w_{1}, w_{2}\right)$ are determined and presented in Figs. 2-7.
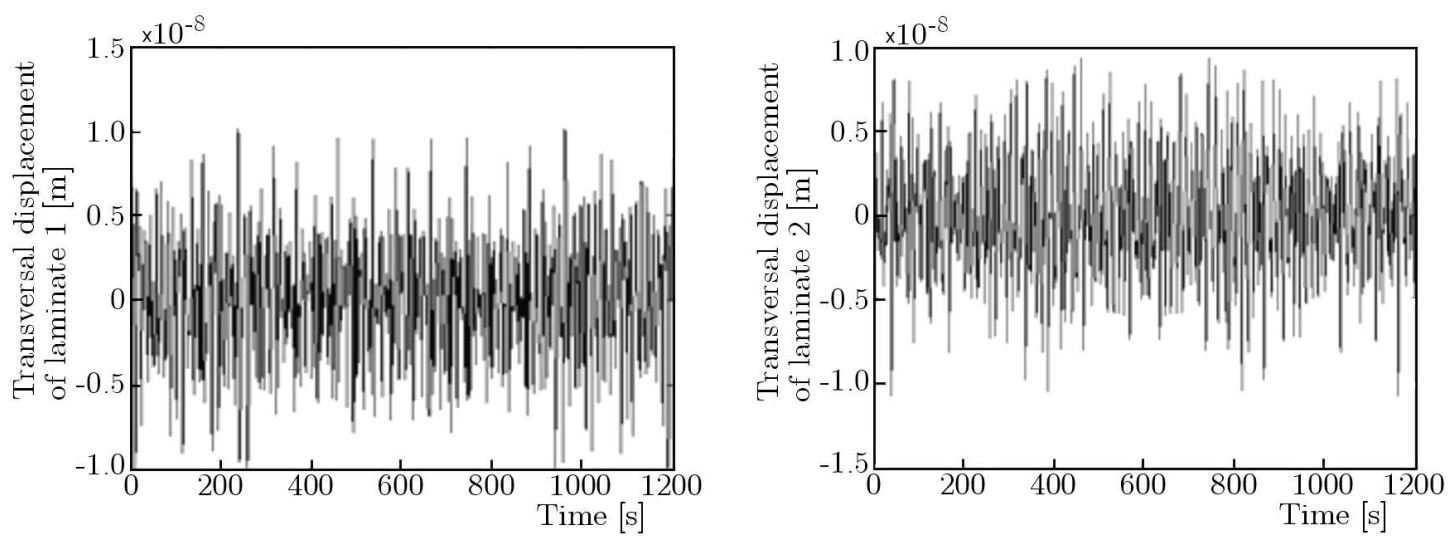

Fig. 2. Model 1: observed signals for a punctual force 

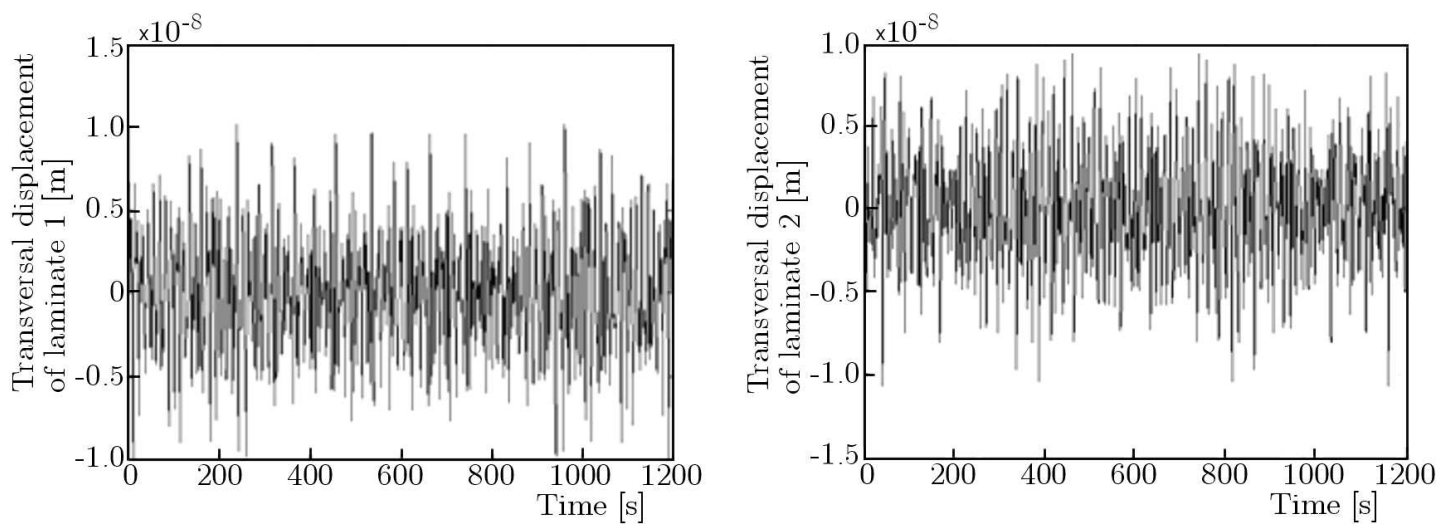

Fig. 3. Model 1: observed signals for a distributed force
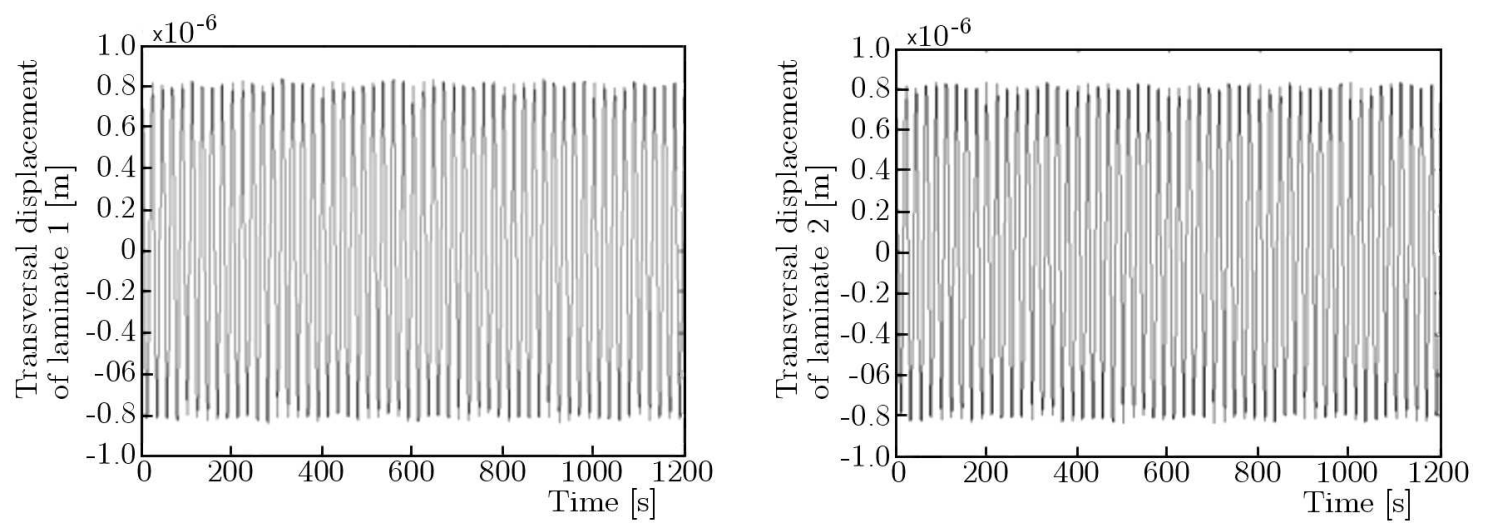

Fig. 4. Model 2: observed signals for a punctual force
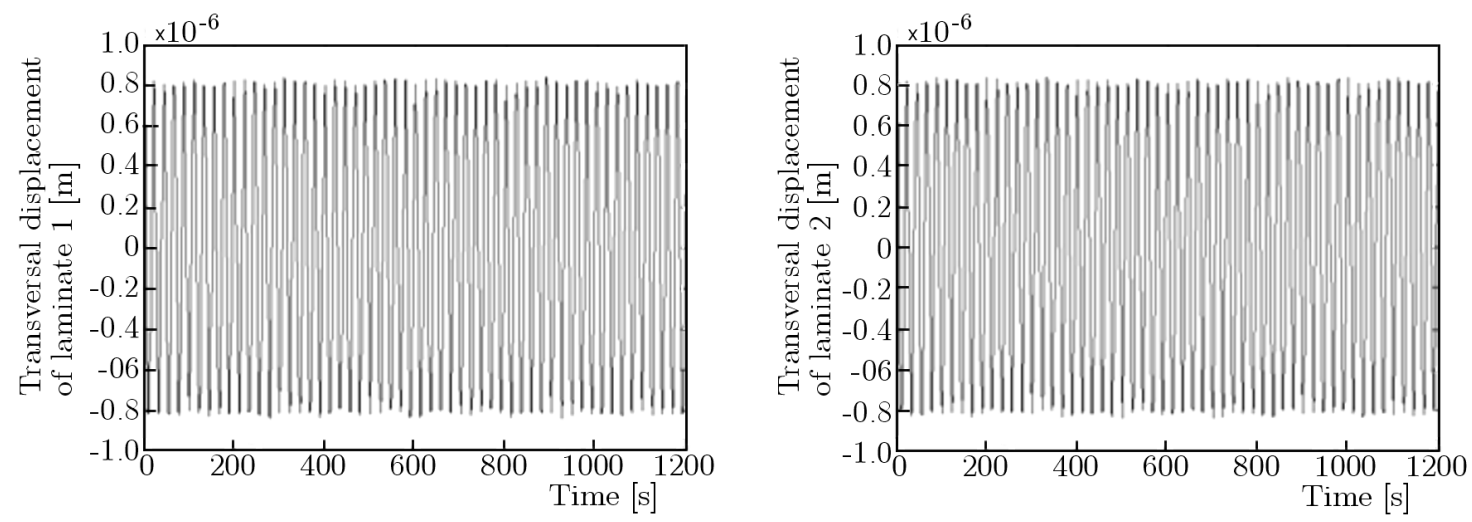

Fig. 5. Model 2: observed signals for a distributed force
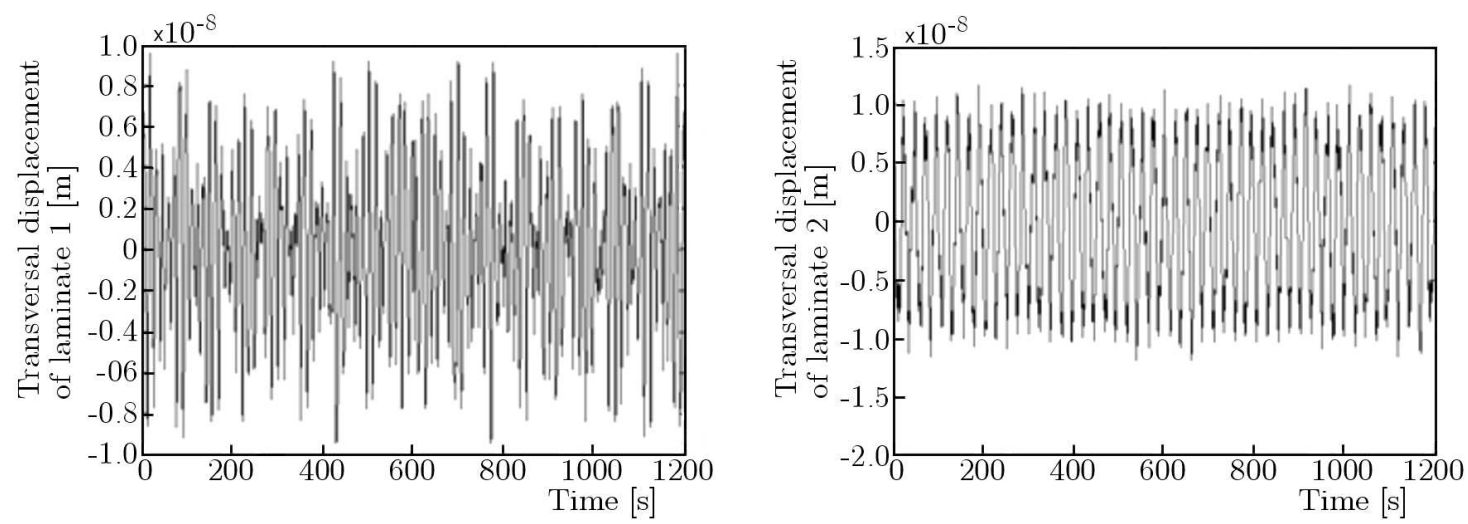

Fig. 6. Model 3: observed signals for a punctual force 

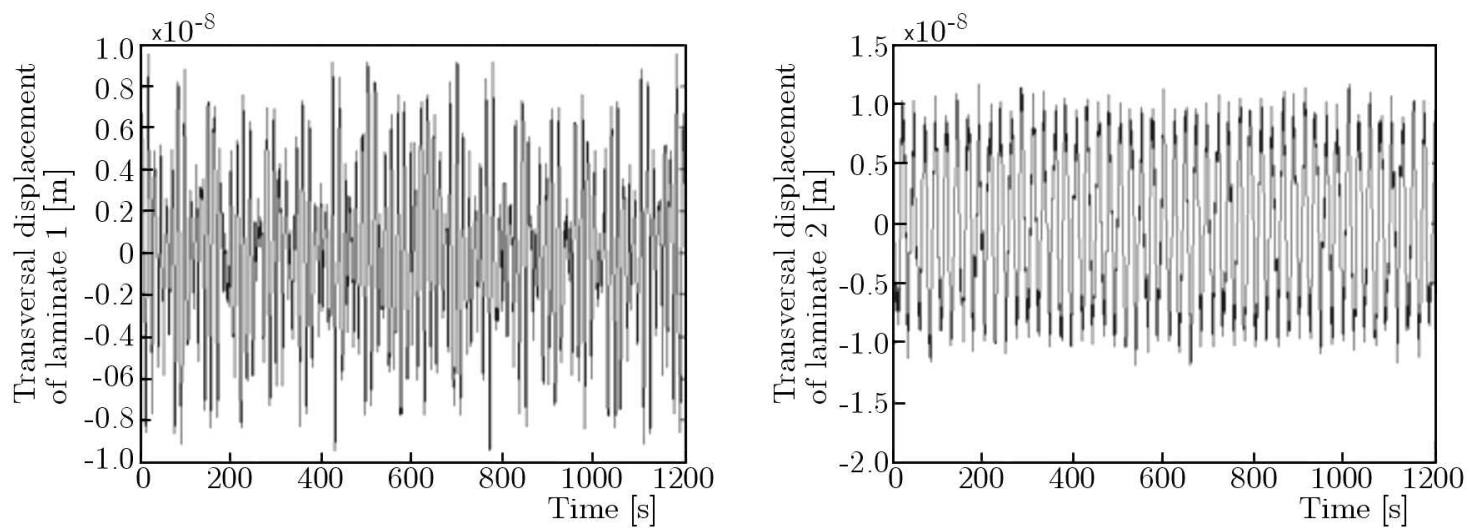

Fig. 7. Model 3: observed signals for a distributed force

Then, after determining the observed signals which represent the displacements in the centre of the first and the second laminate, the obtained results will serve as the input in the ICA algorithm.

\section{3. $\quad$ Estimated sources}

The wave form and excitation frequency of the punctual and distributed forces applied to the system will be constructed using the ICA algorithm.

The time-evolution of the applied force and the corresponding spectrum (FFT) are presented in Figs. 8a and 8b, respectively. In this case, we have mentioned that this applied excitation can be distributed on the whole nodes of laminate 1 . So, it can be also reconstituted by the developed inverse method.

Then, from Fig. 9 to Fig. 14, we present the corresponding ICA results.
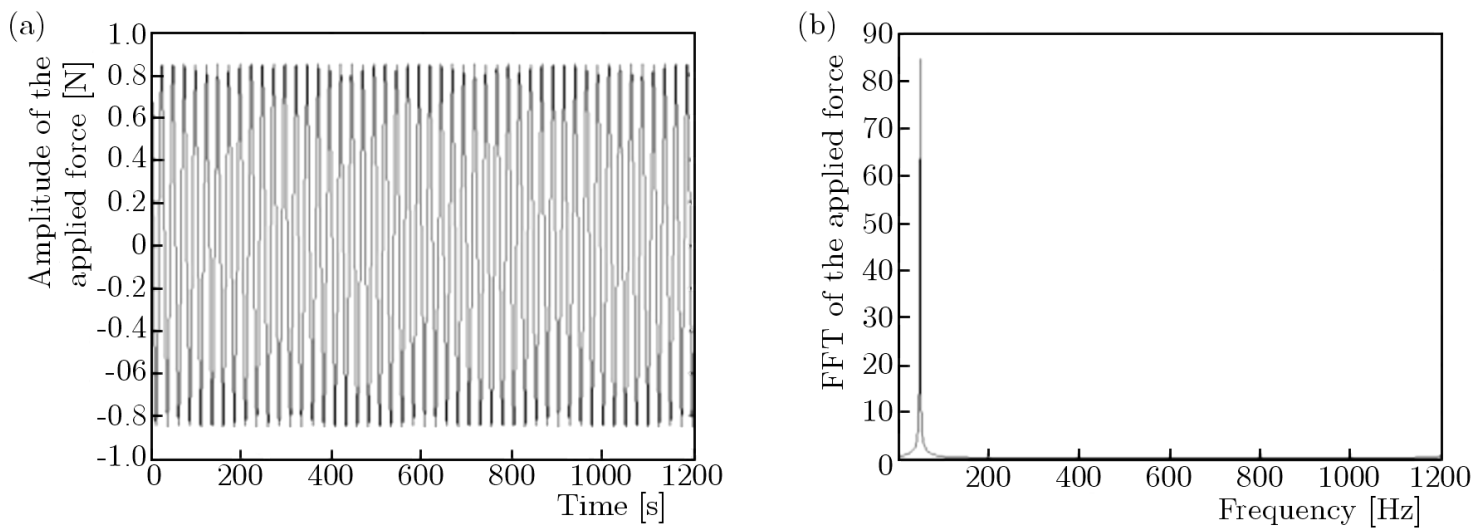

Fig. 8. (a) Time-evolution of the applied excitation source, (b) FFT of the applied excitation source

From Fig. 9 to Fig. 14, we can deduce that the adopted inverse method can be applied to identify the dynamic excitation (the punctual and distributed forces and their spectrum) for analyzing the vibro-acoustic behaviour of a fluid-structure coupled system. In this case, the excitation frequencies of each force are localized and determined.

\section{Conclusions}

In this paper, one of the major techniques of the Blind Source Separation (BSS) called the Independents Components Analysis (ICA) is presented and exploited in order to extract the 

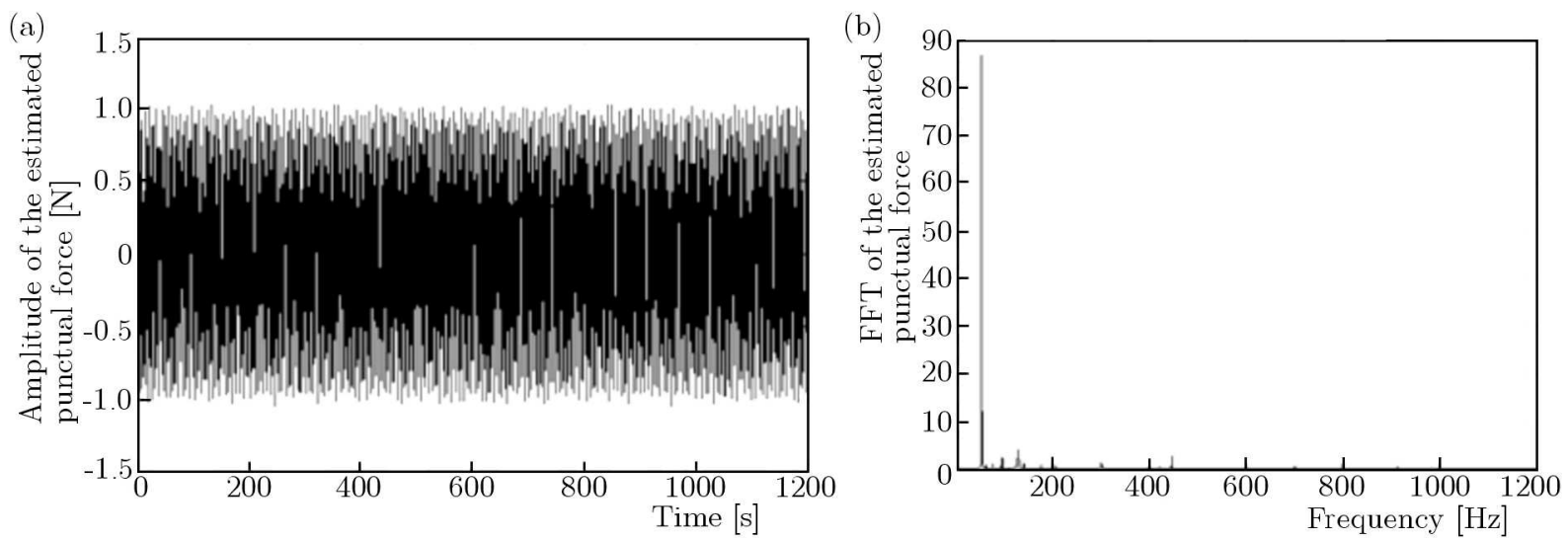

Fig. 9. Model 1: (a) estimated punctual source, (b) FFT of the estimated punctual source
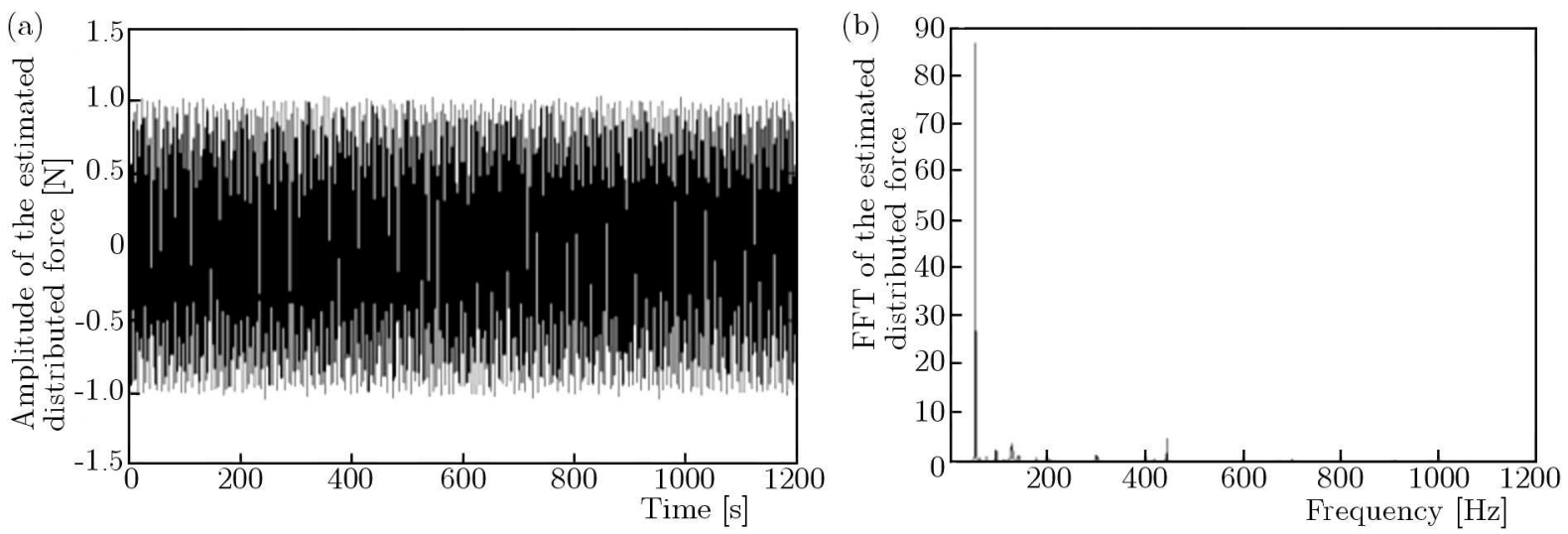

Fig. 10. Model 1: (a) estimated distributed source, (b) FFT of the estimated distributed source
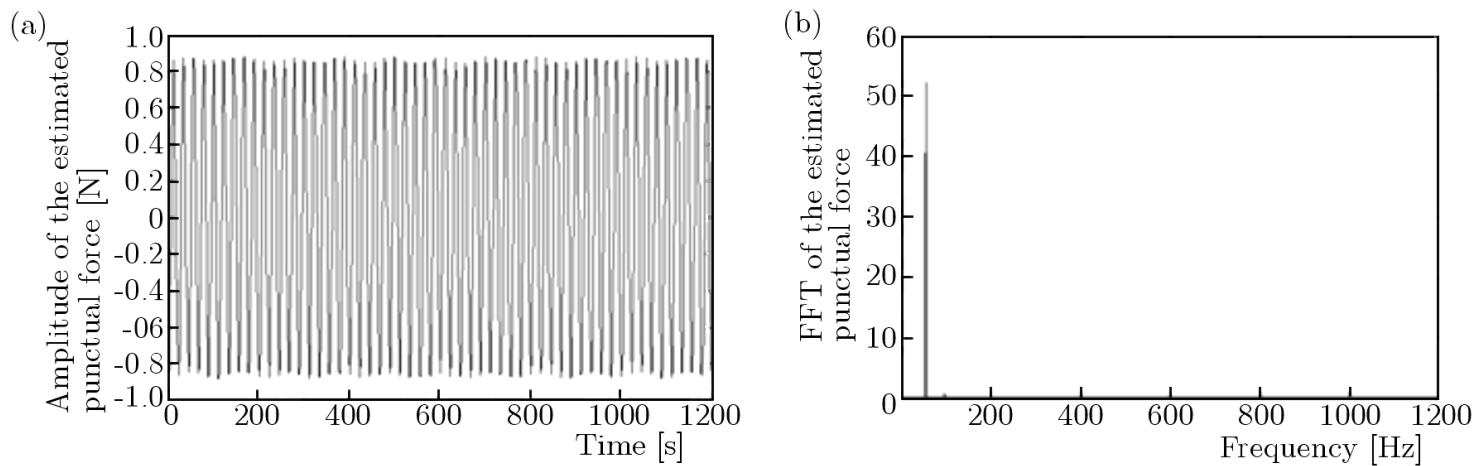

Fig. 11. Model 2: (a) estimated punctual source, (b) FFT of the estimated punctual source
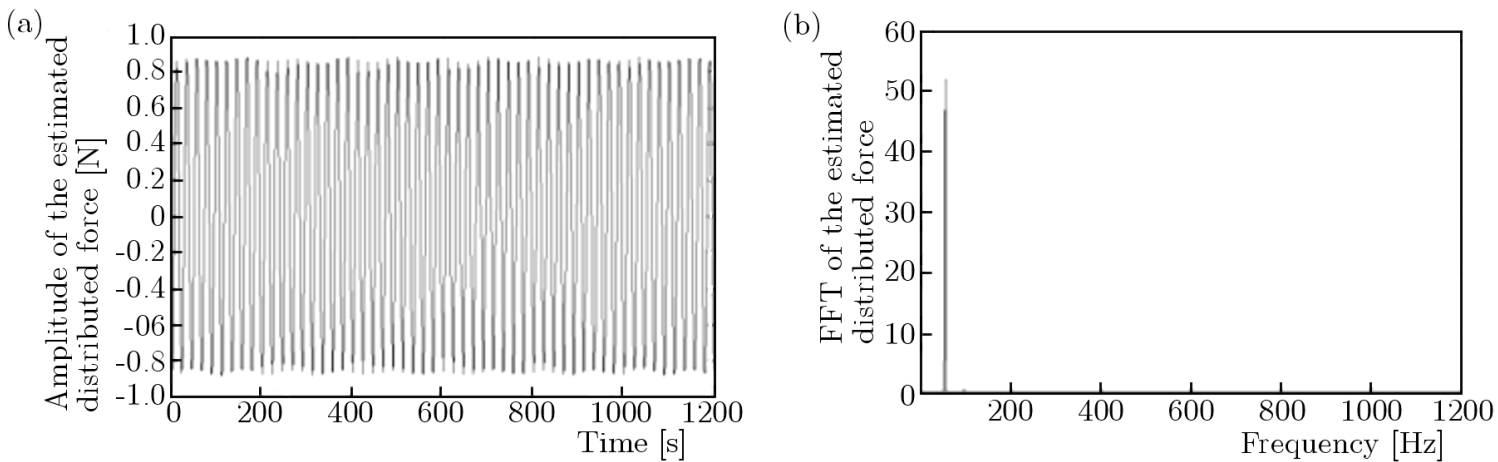

Fig. 12. Model 2: (a) estimated distributed source, (b) FFT of the estimated distributed source 
(a)

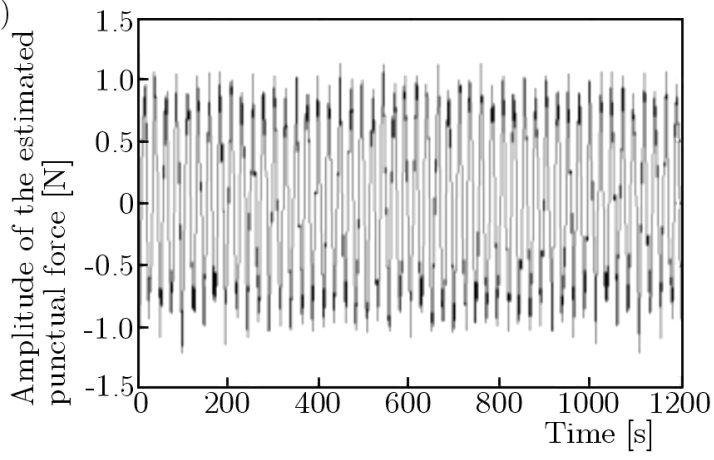

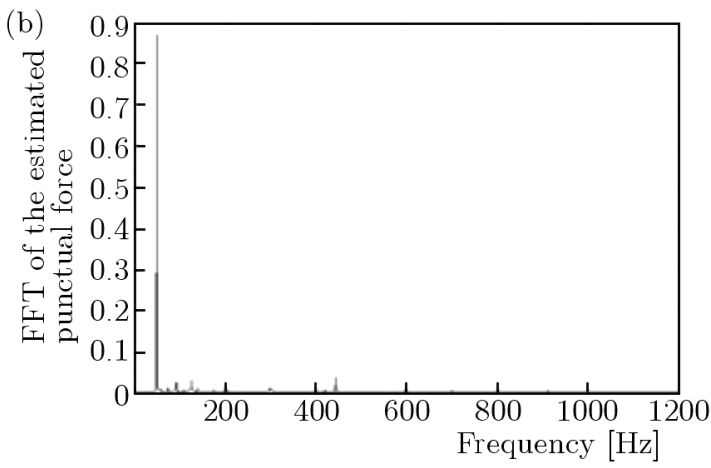

, (b) FFT of the estimated punctual source (a)

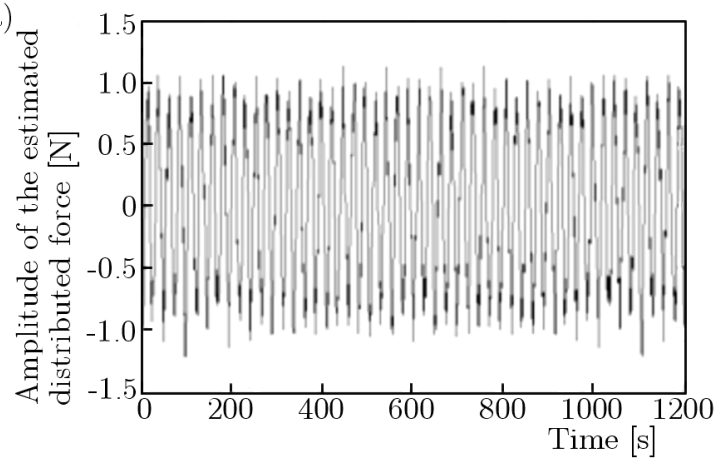

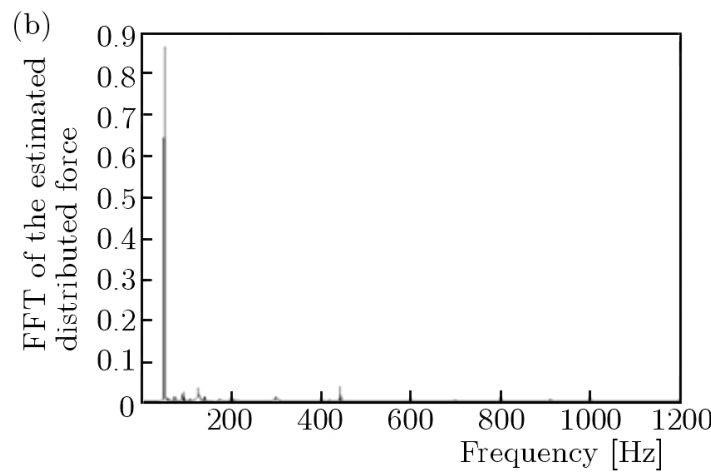

Fig. 14. Model 3: (a) estimated distributed source, (b) FFT of the estimated distributed source

force applied to a fluid-structure coupled system composed of a thin-film laminated double glazing system enclosing a thin fluid cavity and limited by an elastic joint. The vibro-acoustic responses are determined by the modal recombination method which is applied to the Finite Element (FE) coupled matrix system.

As a continuation of our previous published works, this method allows us to determine the wave form of any external or internal forces applied to the structure, so it is a useful method to study complex fluid-structure coupled systems.

\section{References}

1. Abbès M.S., Miladi Chaabane M., Akrout A., Fakhfakh T., Haddar M., 2011, Vibratory behavior of a double panel system by the operational modal analysis, International Journal of Modelling Simulation and Scientific Computing, 2, 4, 459-479

2. Abdennadher M., Fenina S., Hammami L., Haddar M., 2005, Vibro-acoustic analysis of a double sandwich panels system, International Journal of Engineering Simulation, 6, 1, 3-9

3. Akrout A., Hammami L., Karra C., Ben Tahar M., Haddar M., 2010, Vibro-acoustic damping simulation of two laminated glass panels coupled to viscothermal fluid layer, International Journal of Acoustics and Vibration, 15, 2, 79-90

4. Akrout A., Miladi Chaabane M., Hammami L., Haddar M., 2012a, Edge stiffness effects on thin-film laminated double glazing system dynamical behavior by the operational modal analysis, Journal of Mechanics of Materials and Structures, 7, 8/9, 837-859

5. Akrout A., Tounsi D., Taktak M., Abbès M. S., Haddar M., 2012b, Estimation of dynamic system's excitation forces by the independent component analysis, International Journal of Applied Mechanics, 4, 3, 1250032 
6. Antoni J., 2005, Blind separation of vibration components: principles and demonstration, Mechanical Systems and Signal Processing, 19, 1166-1180

7. Cheng L., Li Y.Y., Gao J.X., 2005, Energy transmission in a mechanically linked double-wall structure coupled to an acoustic enclosure, Journal of the Acoustical Society of America, 117, 5, 2742-2751

8. Comon P., 1994, Independent component analysis. A new concept? Signal Processing, 287-314

9. Hammami L., Zghal B., Fakhfakh T., Haddar M., 2005, Modal approach for dynamical analysis of sandwich plates, International Journal of Computer Applications in Technology, 24, 4, 203-211

10. HÉrault J., Ans B., 1984, Réseaux de neurones à synapses modifiables: Décodage de messages sensoriels composites par un apprentissage non supervise et permanent, Comptes Rendus De l'Académie des Sciences, série III, 525-528

11. Hérault J., Jutten C., Ans B., 1985, Détection de grandeurs primitives dans un message composite par une architecture de calcul neuromimétique en apprentissage non supervise, Actes du $\boldsymbol{X} \underline{\text { eme }}$ colloque GRETSI, Nice, France, 1017-1022

12. HyväRInEN A., OJA E., 2000, Independent component analysis: algorithms and applications, IEEE Transactions on Neural Networks, 13, 4/5, 411-430

13. Moreau E., Macchi O., 1993, New self-adaptive algorithms for source separation based on contrast functions, IEEE Signal Processing Workshop on Higher-Order Statistics, South Lac Tahoe, USA (CA), 215-219

14. Taktak M., Tounsi D., Akrout A., Abbès M. S., Haddar M., 2012, One stage spur gear transmission crankcase diagnosis using the independent components method, International Journal of Vehicle Noise and Vibration, 8, 4, 387-400

15. Zarzoso V., Comon P., 2008, Robust independent component analysis for blind source separation and extraction with application in electrocardiography, 30th Annual International IEEE EMBS Conference, Vancouver, British Columbia, Canada

16. Zarzoso V., Comon P., 2010, Robust independent component analysis by iterative maximization of the Kurtosis contrast with algebraic optimal step size, IEEE Transactions on Neural Networks, 21 